Revue musicale OICRM

revue musicale oicrm

\title{
Peindre, écouter, écrire, de Rita Ezrati et Jean-Jacques Nattiez
}

\section{Dujka Smoje}

Volume 5, numéro 2, 2018

URI : https://id.erudit.org/iderudit/1054152ar

DOI : https://doi.org/10.7202/1054152ar

Aller au sommaire du numéro

Éditeur(s)

Observatoire interdisciplinaire de création et recherche en musique (OICRM)

ISSN

2368-7061 (numérique)

Découvrir la revue

Citer ce compte rendu

Smoje, D. (2018). Compte rendu de [Peindre, écouter, écrire, de Rita Ezrati et Jean-Jacques Nattiez]. Revue musicale OICRM, 5(2), 180-186.

https://doi.org/10.7202/1054152ar

Ce document est protégé par la loi sur le droit d'auteur. L'utilisation des services d'Érudit (y compris la reproduction) est assujettie à sa politique d'utilisation que vous pouvez consulter en ligne.

https://apropos.erudit.org/fr/usagers/politique-dutilisation/
Cet article est diffusé et préservé par Érudit.

Érudit est un consortium interuniversitaire sans but lucratif composé de l’Université de Montréal, l'Université Laval et l'Université du Québec à Montréal. Il a pour mission la promotion et la valorisation de la recherche. https://www.erudit.org/fr/ 


\title{
Peindre, écouter, écrire, de Rita Ezrati et Jean-Jacques Nattiez Sampzon, Delatour France, 2017, 93 pages
}

\author{
Dujka Smoje
}

Mots clés : relations peinture/musique; iconographie musicale; perception esthétique.

Keywords: relationship painting/music; musical iconography; aesthetic perception.

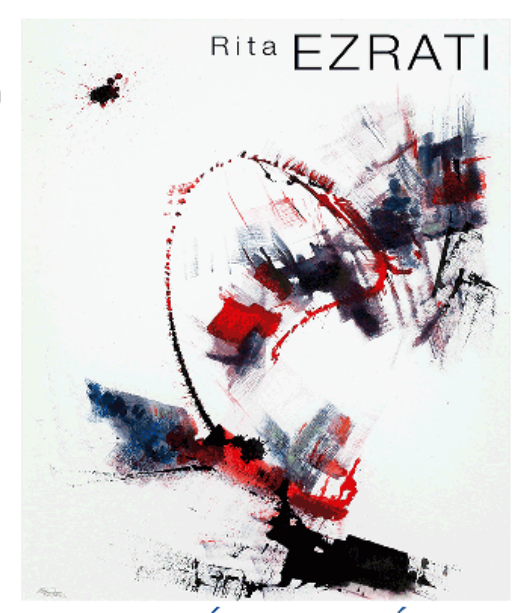

Peindre Écouter Écrire DEAATOUR Jean-Jacques Nattiez
C'est un livre composé à quatre mains, pinceaux plume complices ; la musique en prime. Un plaisir pour les yeux et pour l'esprit. Lorsqu'on a fait le tour des images et parcouru les textes, une question s'impose : peut-on décrire un art au moyen d'un autre ; la musique peut-elle être dépeinte par la peinture? Même si on y ajoute un commentaire ou une analyse?

Les soixante-six œuvres de Rita Ezrati, huiles et encres, reproduites avec une grande fidélité, offrent un éventail d'inspirations visuelles, qu'accompagnent trente-trois textes de Jean-Jacques Nattiez. Il s'agit de commentaires et de notes de programme, présentant des œuvres musicales, pour la plupart récentes. Chacun des deux auteurs explique sa part dans ce jeu à quatre mains. Un témoin, le philosophe Georges Leroux, ajoute un essai : il survole les deux répertoires qui se répondent dans ce livre et nous offre sa vision très personnelle sur la possibilité de rencontres entre peinture et musique, dans « un langage commun, celui du contrepoint des formes » (p. 19). 
Lorsqu'on aborde les projets qui cherchent à établir une correspondance entre peinture et musique, les artistes partent le plus souvent d'un modèle musical pour concevoir leur œuvre picturale. La démarche Ezrati/Nattiez suit deux approches différentes : d'une part, la majorité des liens proviennent d'une rencontre entre des toiles déjà existantes et des compositions choisies dans le répertoire traité par le musicologue, selon l'intuition de l'artiste. D'autre part, quelques huiles et encres ont été directement inspirées par l'écoute de certaines œuvres musicales, telles les planches dédiées à Serge Garant (p. 46-51), ou encore les compositions de Boulez, Sokolović, Vacchi (p. 62-65, 58-61, 73-85). La page de présentation de Rita Ezrati indique comment elle a conçu et organisé ce contrepoint en toute indépendance du texte de son complice.

La plupart des images aux couleurs intenses sont des reproductions de toiles non figuratives, sinon à la limite de l'abstraction. Rarement, une figure se glisse discrètement dans le champ des couleurs, davantage suggestion qu'illustration (Tsunami, 2015, p. 12 ; Dans la tourmente, 2016, p. 80 ; Le rêve du Masque, 2015, p. 24, figure 1).

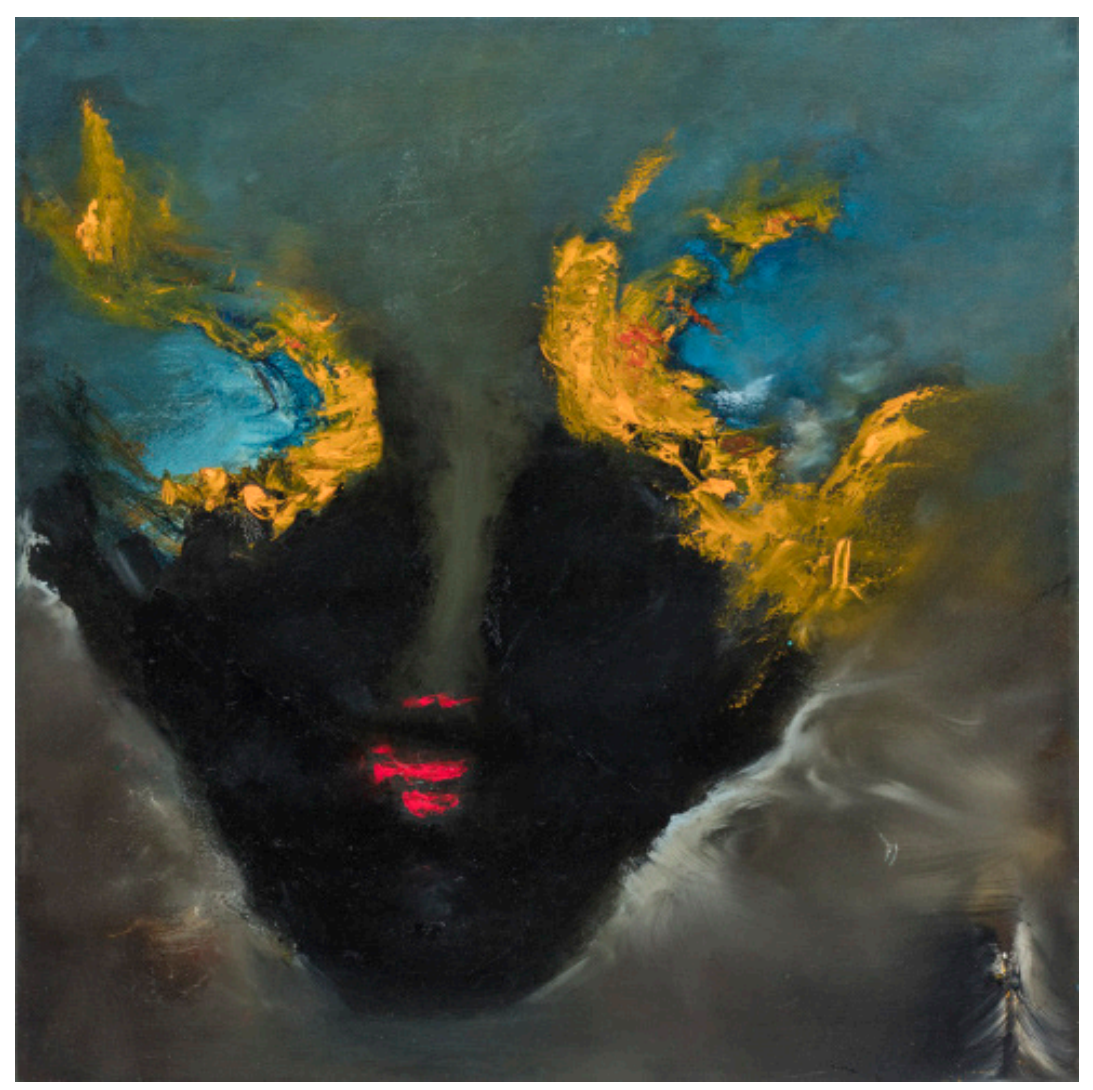

Figure 1: Le rêve du Masque, 2015, p. 24. 


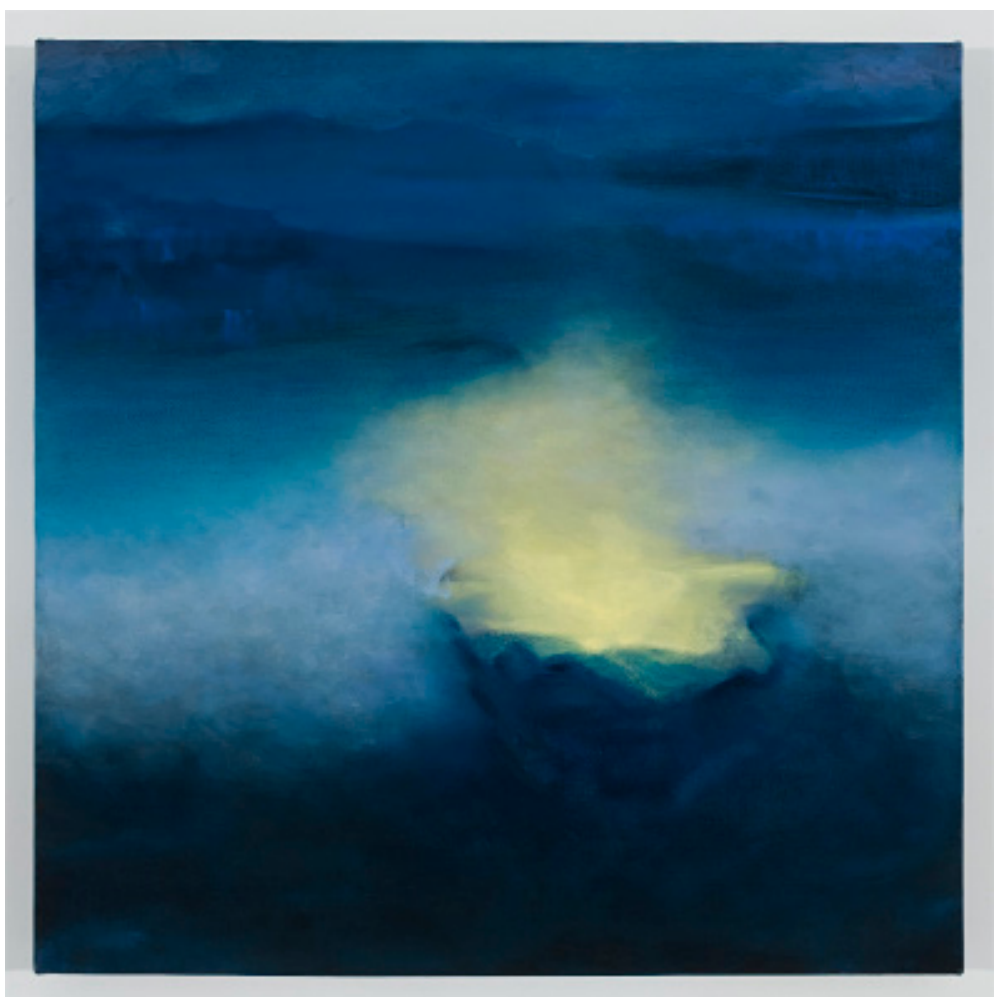

Figure 2 : Éveil, 2010, p. 52 ; tableau associé au solo de cor anglais de Tristan et Isolde (1858) de Richard Wagner.

Dans les profondeurs du champ de bleu marine jaillit une lumière irréelle, éclatant dans l'ombre dense. L'horizon semble sans limites et le regard dépasse le cadre visible de la toile. Le spectateur a l'impression de se perdre dans l'immensité de l'océan.

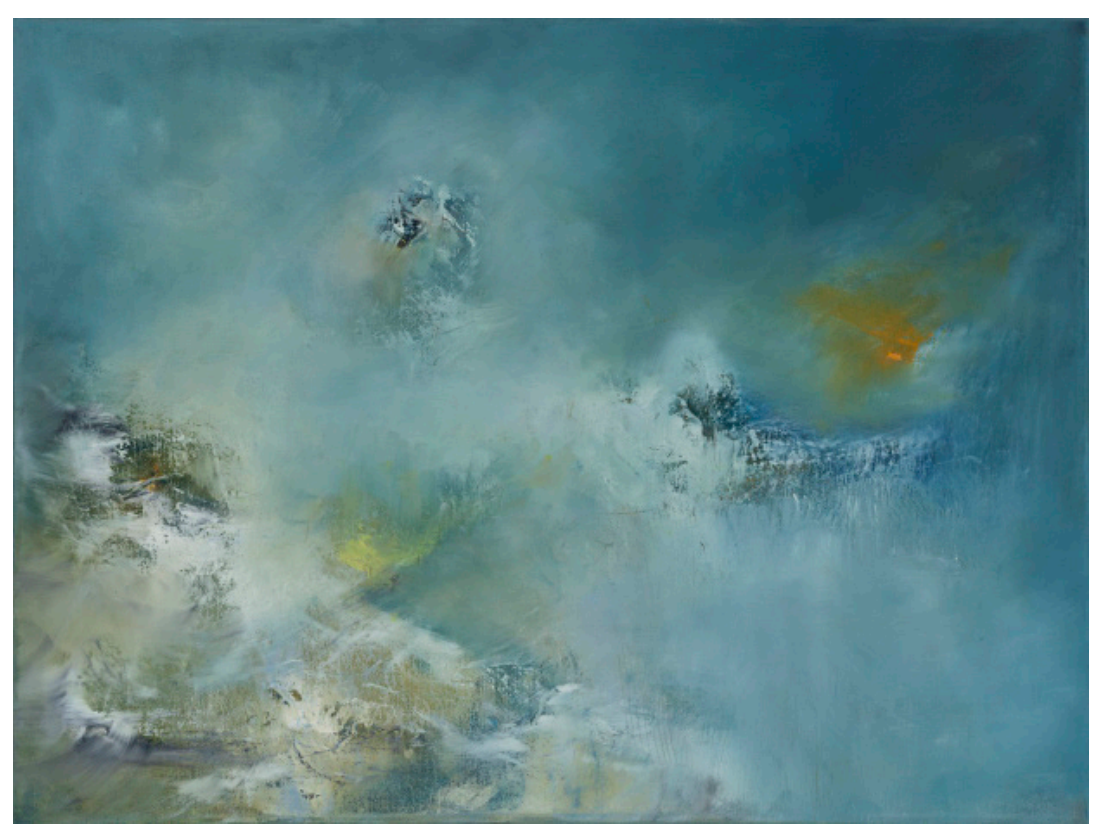

Figure 3 : Embrumée, 2014, p. 34-35; tableau associé à In un silenzio ordinato (1985) de Ada Gentile.

Dans cette toile en camaïeu turquoise, l'espace est animé par des îlots de brouillard gris et blancs. On a l'impression de flotter en douceur dans les nuages, comme en apesanteur, dans un rêve. 


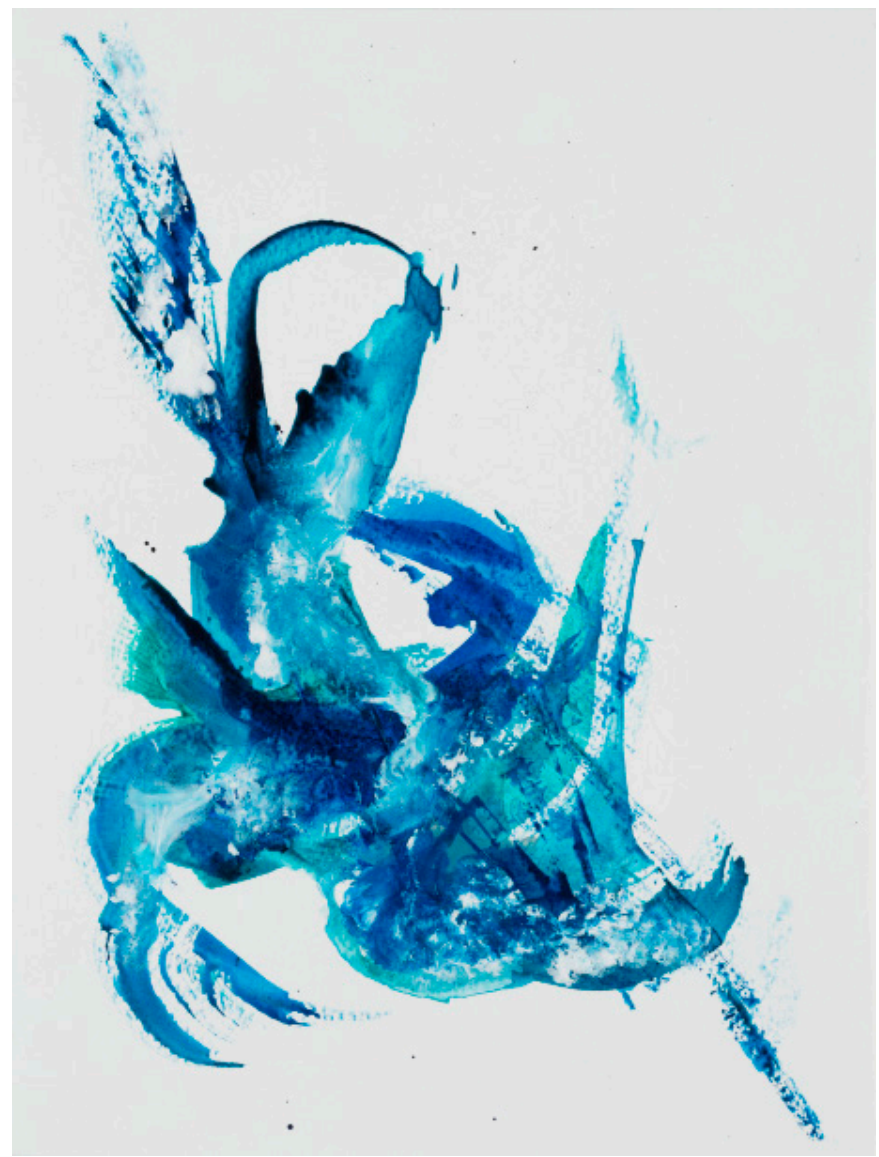

Figure 4: Le printemps... bientôt, 2016, p. 16.

Ici, une arabesque en bleu et ses lignes dansantes ; la grâce des glissandi de couleur rappelle la calligraphie japonaise.

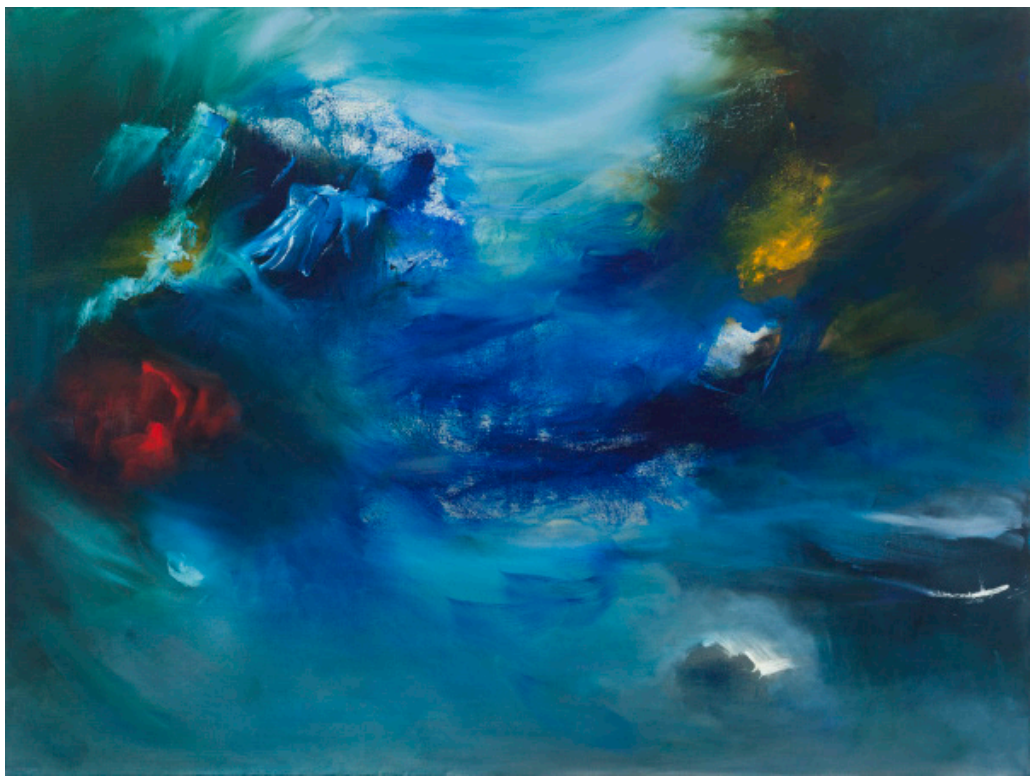

Figure 5 : Lame de fond, 2015, p. 8.

$\mathrm{Au}$ milieu de la turbulence aquatique, l'éruption sous-marine libère les éclats de lumière rouge, jaune et blanche. L'intensité des couleurs crée un conflit dramatique 
entre les nuances de bleu du fond et les invasions du noir, au cœur d'une scène imaginaire en trois dimensions.

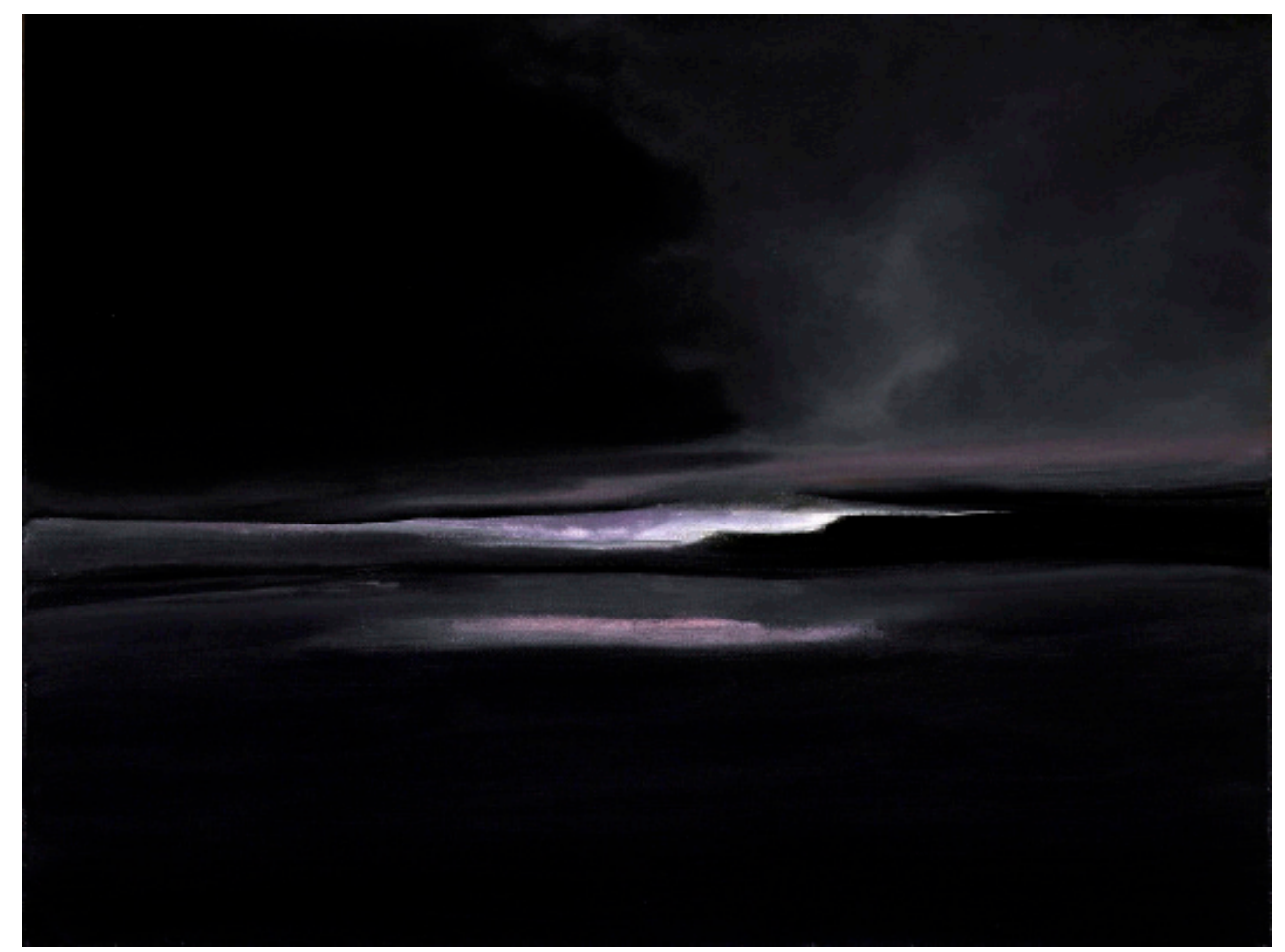

Figure 6: Promesse d'infini, 2008, p. 54 ; tableau associé à La cathédrale engloutie (1910), prélude pour piano de Claude Debussy.

Dans un noir profond illimité, l'horizon semble déchiré par deux traits de lumière, blanc et gris. Des reflets de couleur noire animent la toile monochrome dont l'intensité invite à l'intériorité et à la méditation. Une pensée pour Soulages.

Malgré les titres, la rupture avec le figuratif est évidente. Le regard suit le jeu d'ombre et de lumière, suggérant une troisième dimension, d'où émergent les formes et les couleurs, reflets des paysages intérieurs de l'artiste.

Quelles émotions accompagnent le regard qui plonge dans ces toiles ? Comme celles que la musique pure fait naître, elles viennent de cette zone inaccessible à la conscience éveillée. Ce sont des créations d'une énergie dont le courant se transmet spontanément, libérant la dynamique des couleurs et des formes, dont on sent les vibrations longtemps après avoir détaché le regard de l'image.

Pour vraiment entrer dans cet univers de correspondances et jouer le jeu jusqu'au bout, il faudrait pouvoir écouter les œuvres musicales choisies par Jean-Jacques Nattiez tout en regardant les toiles de Rita Ezrati. On comprend que pour des raisons techniques et financières ce ne soit pas possible. Par chance, Internet offre une solution au lecteur intéressé, qui peut écouter certaines œuvres sur YouTube ou iTunes. D'autres, plus récentes, de compositeurs plus jeunes et moins connus, ne sont accessibles que sur disques dont on trouve les références à la fin du livre. 
Les textes de Nattiez, destinés au grand public, présentent un éventail d'œuvres musicales couvrant deux siècles, de Beethoven, Wagner et Debussy jusqu'à Ana Sokolović (2014). Ils apportent des informations et des commentaires très utiles, insistant sur la perception et l'expérience auditive subjective. La curiosité du lecteur se trouve éveillée ; il aimerait devenir auditeur, surtout lorsqu'il s'agit d'œuvre récentes, encore inconnues, peu jouées ou difficilement accessibles. Certaines remarques révèlent des préférences personnelles; à travers (ou malgré ?) des observations factuelles ou analytiques sur les compositions contemporaines se glisse une touche poétique, qui rapproche l'intellectuel de l'artiste.

Des exemples? Impossible de citer quelques phrases isolées ; il faut lire l'ensemble de chaque note de programme, et si possible, écouter la musique pour capter les fils croisés entre le texte, l'image, le son. Mes préférences :

- Linda Bouchard, Les musique du feu, de la terre et de l'eau (1993-1996), p. 40-41.

- Ada Gentile, In un silenzio ordinato (1985), p. 35.

- Fabio Vacchi, Voci di notte (2006), p. 85.

- Francis Dhomont, Sous le regard d'un soleil noir (1979-1981), p. 43-44.

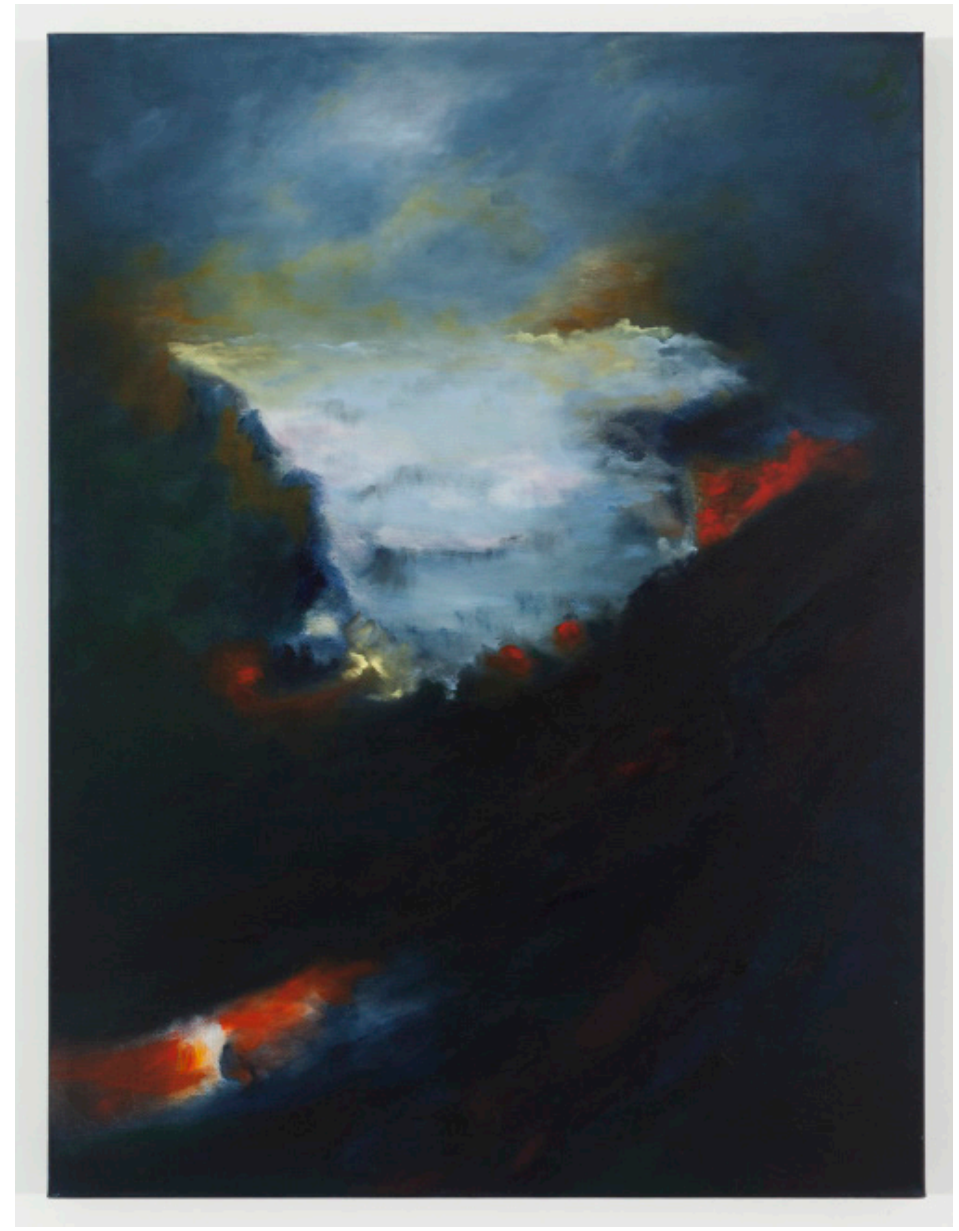

Figure 7 : Dante, 2011, p. 85 ; tableau associé à Voci di notte (2006) de Fabio Vacchi. 
Enfin, revenons à la question de départ : peut-on décrire un art au moyen d'un autre? La musique peut-elle évoquer la peinture ou la peinture faire écho à la musique?

La réponse est ambiguë. Lorsqu'il s'agit d'une métaphore ou d'une évocation intuitive, on reste au niveau de la perception subjective qui est rarement partagée, variant d'une personne à l'autre. Les perceptions multiples ouvrent l'éventail très large aux rencontres croisées du visuel, de l'écrit et du sonore. Y a-t-il convergence? La suggestion de rapprochement peut guider le spectateur-auditeur, révéler la sensibilité ou la source d'inspiration de l'artiste. Cependant demeure toujours la différence entre l'œuvre conçue et l'œuvre perçue. Mais si la correspondance ne peut être démontrée, le charme de la liaison demeure.

La description objective devient possible lorsque le peintre suit la stratégie compositionnelle de la musique pour construire l'œuvre picturale, ainsi qu'en témoignent les fugues sur toile de Klee ou de Kupka ${ }^{1}$, ainsi que les contrepoints sur vitrail de van Doesburg $^{2}$ qui ont marqué le passage de l'art figuratif vers l'art abstrait. Ou encore, les Farbsymphonien ${ }^{3}$ du peintre suisse Jakob Weder, à la fin du siècle dernier, qui transposent à la lettre des partitions de Bach, appliquant sur la toile les couleurs en camaïeu avec une précision mathématique. L'effet visuel est saisissant. Cependant, si le spectateur ne le sait pas, rien ne lui dit qu'il s'agit de la musique devenue peinture.

On l'aura compris, en l'occurrence cette stratégie n'est pas celle de Rita Ezrati. Ses peintures, subtilement associées à la musique, créent un univers inaccessible à notre pensée rationnelle. Pour les décrire, il faudrait une autre œuvre d'art - un poème - qui serait leur équivalent, qui aurait le pouvoir de susciter une émotion semblable, intense et ineffable.

1 Paul Klee, Fugue en rouge (1921), Croissance des plantes (1921), František Kupka, Amorpha - Fugue en deux couleurs (1912). Tous les sites internet ont été consultés le 15 mars 2018.

2 Theo van Doesburg, Stained-glass Composition IV (1917). Au sujet de la structure musicale du vitrail d'après Bach, Offrande musicale, Canon cancrizans, dans Friedrich Teja Bach, " Johann Sebastian Bach in der klassischen Moderne ", dans Karin von Maur (éd.), Vom Klang der Bilder, Die Musik in der Kunst des 20. Jahrhunderts, Munich, Prestel, 1985, p. 328-334.

3 Jakob Weder, Farbsymphonien (1980-1990), Jean-Sébastien Bach, Troisième Suite pour orchestre, BWV 1068. Explication détaillée dans Dujka Smoje, "Colors of Bach's Music. Farbsymphonien in Jakob Weder's Painting ", Music in Art, vol. XXIX, nos 1-2, 2004, p. 265-272, https://www.academia. edu/5788154/Colors of Bachs Music Farbsymphonien in Jakob Weders Painting. 